\title{
Editorial
}

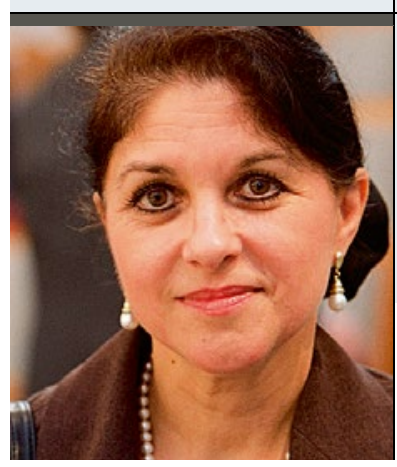

"Durch ehrenamtlich organisierte Ärzte gelingt zwar eine Minimalversorgung, jedoch keine flächendeckende medizinische Versorgung."

\section{Flüchtlinge: Hier besteht dringender Handlungsbedarf!}

W ir erleben zurzeit eine in den letzten Jahrzehnten nicht da gewesene Fluchtbewegung. Laut dem Flüchtlingswerk der Vereinten Nationen (UNHCR) befinden sich weltweit etwa 60 Millionen Menschen auf der Flucht [1]. Diese Welle hat auch Deutschland erreicht. Bei den Top-Ten der Länder, aus denen die Geflüchteten stammen, steht im Zeitraum Januar bis Oktober 2015 Syrien an erster Stelle, gefolgt von Balkanstaaten, Afghanistan und Irak [2]. Die Zahl der in Deutschland bis Oktober 2015 gestellten Asylanträge wird mit etwa 332.000 erhoben, im Vergleich dazu betrug diese Zahl für das gesamte Jahr 2014 202.834 [2]. Wie hoch die Zahl der Geflüchteten in Deutschland inzwischen tatsächlich ist, lässt sich nicht angeben, da ein nicht unerheblicher Anteil der Flüchtlinge noch nicht registriert werden konnte.

Flüchtlinge sind vorwiegend Menschen, die aufgrund von Krieg, sozialer oder politischer Instabilität und/oder anderer Konflikte Opfer von körperlicher, sexueller und/oder psychologischer Gewalt geworden sind [3]. Die meisten Flüchtlinge sind bei ihrer Ankunft in Deutschland in einem sehr schlechten körperlichen und psychischen Zustand [4], völlig erschöpft und ausgelaugt. Die prekären Aufnahmebedingungen in Gemeinschaftsunterkünften mit sehr beengten Wohnmöglichkeiten, ohne Privatsphäre und soziale Unterstützung stellen Postmigrationsstressoren dar, die ebenfalls zu einer nachhaltigen Beeinträchtigung der psychischen Gesundheit führen können.

Vor diesem Hintergrund ist es irritierend, dass die akute medizinische Versorgung vielerorts nicht gesichert erscheint. Gerade der erschwerte Zugang zum Gesundheitssystem geht mit einer weiteren Verschlechterung der bereits vorliegenden psychischen Störungen einher [5]. Denn das Asylbewerberleistungsgesetz bestimmt, dass dem Personenkreis der Asylbewerber nur in akuten Krankheits- situationen wie Krisen, Suizidalität oder akuten Schmerzzuständen mit einem grünen Krankenschein vom Sozialamt eine Behandlung gewährt wird [6]. Für chronische Erkrankungen sind medizinische Behandlungen nicht vorgesehen. Leistungen wie stationäre Behandlung und häusliche Krankenpflege sind damit nicht abgedeckt und müssen extra durch das Sozialamt genehmigt werden [6].

Nach 15 Monaten erhalten Asylbewerber eine Versichertenkarte der GKV, mit der sie den einheimischen Patienten gleichgestellt sind. Die hier entstehenden Kosten werden über die Sozialbehörden refinanziert, so dass es sich hier um keine echte Kassenleistung handelt [6]. Mit der Versichertenkarte kann der Asylbewerber die medizinische Versorgung direkt in Anspruch nehmen. In Bremen und Hamburg erhalten die Flüchtlinge bereits bei der Registrierung eine Gesundheitskarte.

\section{Hauptzugangsbarriere ist die Sprache}

Damit sind jedoch nicht alle Zugangsbarrieren beseitigt. Oftmals ist nämlich der Zugang durch sprachliche und kulturelle Verständigungsprobleme erheblich erschwert, so dass Missverständnisse zu Fehldiagnosen und Fehlbehandlungen [7] sowie unnötigen Kosten [8] führen. Diese könnten durch den Einsatz von qualifizierten Sprach- und Kulturmittlern reduziert werden, die jedoch nicht flächendeckend zur Verfügung stehen. Für die Kosten gibt es bislang keine Finanzierungssicherheit. Damit wird die Sprachbarriere, eine der Hauptzugangsbarrieren nicht beseitigt, obwohl die Sprache das Hauptarbeitsinstrument der Psychiatrie und Psychotherapie ist $[9,10]$. Nach Lindert et al. [11] leiden etwa $44 \%$ der Flüchtlinge und Asylwerber unter einer Depression, $40 \%$ unter einer Angststörung und $36 \%$ unter einer PTBS, während Heeren et al. [12] von $42-85 \%$ mit einer Depression, $39-63 \%$ mit einer Angststörung und 5-54\% mit einer PTBS 


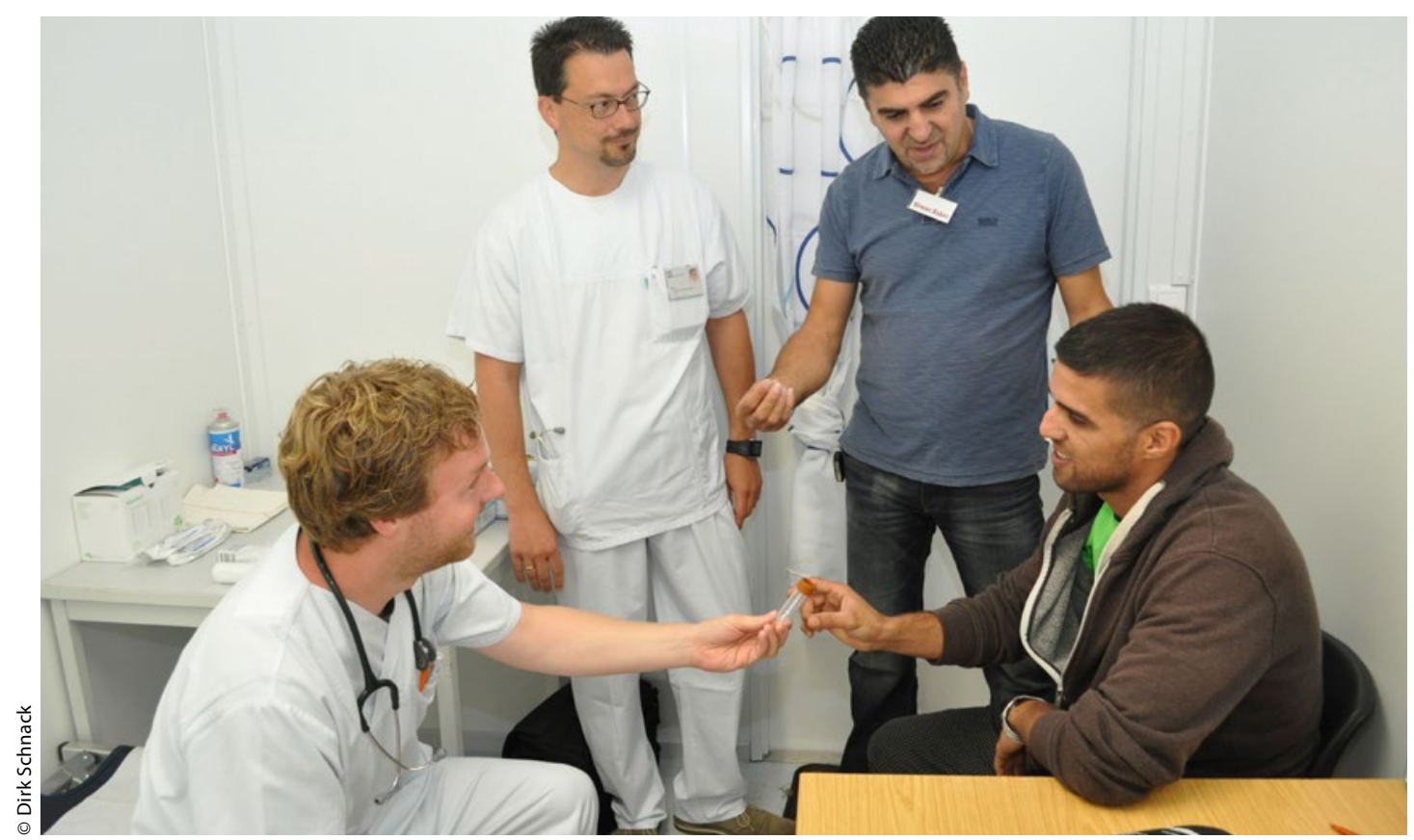

Dr. Alexander Humberg (I.) und Dr. Florian Reifferscheid vom Universitätsklinikum Schleswig-Holstein versorgen in der neuen Flüchtlingsunterkunft in Rendsburg mit Hilfe von Dolmetscher Sirwan Banban einen Patienten aus dem Irak.

ausgehen. Fazel et al. [13] und Crumlish et al. [14] weisen darauf hin, dass die Rate der PTBS bei Flüchtlingen und Asylbewerbern im Vergleich zur Allgemeinbevölkerung um das Zehnfache erhöht ist. Diese hohen Zahlen an psychisch erkrankten Asylbewerbern machen deutlich, dass sich eine große Welle an psychisch sehr belasteten Menschen auf das Gesundheitssystem zubewegt. Die Bundespsychotherapeutenkammer (BPtK) [15] beziffert bei gleichbleibender Versorgungssituation den Anteil der psychisch erkrankten Menschen unter den in diesem Jahr zu erwartenden 800.000 Flüchtlingen, die eine psychotherapeutische Behandlung erhalten können, als unter $1 \%$. Damit wird allein für die psychotherapeutische Behandlung eine große Versorgungslücke offenkundig. Flüchtlinge und Asylbewerber benötigen jedoch primär eine regelhafte medizinische Versorgung, die vielerorts offenbar nur durch ehrenamtlich organisierte Ärzte und Pflege gewährleistet wird. Dadurch gelingt zwar eine Minimalversorgung, jedoch noch keine flächendeckende medizinische Versorgung. Hier besteht dringender Handlungsbedarf.

\section{Mit herzlichen Grüßen}

Ihre

\section{Meryam Schouler-Ocak}

\section{Literatur:}

1. www.unhcr.de

2. www.bamf.de

3. Bhugra et al. European Psychiatry 2014; 29 (2): 107-15

4. Wirtgen W. Dtsch Arztebl 2009; 106 (49): A-2463/B-2115/ C-2055

5. Laban CJ et al. J Nerv Ment Dis 2004; 192: 843-52

6. Frisch J. Berliner Ärzteblatt 2015; 6: 14-20

7. Zaumseil M. in: Transkulturelle Psychiatrie - Interkulturelle Psychotherapie. Interdisziplinäre Theorie und Praxis. 2006; Springer Medizinverlag, Heidelberg, S. 3-50

8. Brucks U, Wahl WB. In: Gut versorgt? Migrantinnen und Migranten im Gesundheits- und Sozialwesen. 2003; Mabuse, Frankfurt, S. 15-34

9. Kluge U. in: Praxis der Interkulturellen Psychiatrie und Psychotherapie. Migration und psychische Gesundheit. 2011; Elsevier, Urban \& Fischer, München, S. 145-54

10. Schouler-Ocak M. Nervenarzt 2015; 86: 1320-5

11. Lindert J et al. Soc Sci Med 2009; 69: 246-57

12. Heeren M et al. Compr Psychiatry 2014; 55 (4): 818-25

13. Fazel M, Wheeler J, Danesh J. The Lancet 2005; 365 (9467): 1309-14

14. Crumlish N, O’Rourke K. J Nervous \& Mental Disease 2010; 198: 4: 237-51

15. BPtK-Standpunkt: Psychische Erkrankungen bei Flüchtlingen, 2015, www.bptk.de

PD Dr. med. Meryam Schouler-Ocak

Psychiatrische Universitätsklinik der Charité im St. Hedwig-Krankenhaus Große Hamburger Str. 5-11, 10115 Berlin E-Mail: meryam.schouler-ocak@charite.de 